\title{
DIE SCHAUKEL IN DER UNGARISCHEN VOLKSTRADITION
}

\author{
Klára GAZDA \\ Universitatea Babeş-Bolyai, Fac. de Filologie Catedra de Limba Maghiara \\ 3400 Cluj-Napoca Str. Horea 31., Rumänien
}

\section{DIE SCHAUKEL ALS SPIELGERÄT}

Die Schaukel ist ein Spielgerät, das bei dem auf ihr Sitzenden ein aus dem Schwingen bzw. Drehen resultierendes besonderes - Schwindel und Benommenheit erregendes, aber letztlich angenehmes - Gefühl erzeugt. Das Kleinkind kann auch ohne Gerät, im Schoß oder auf dem Fuß (sitzend oder stehend) geschaukelt werden. Manchmal schwingen die Größeren ihre Kameraden zweiseitig an den Schultern und Knöcheln haltend. Unter den Gegenständen ist primär die Wiege oder ein ihr vorangehender, sie ersetzender Korb, eine Mulde, ein aufgehängtes Tuch der Liege- und Schlafplatz des noch nicht sitzfähigen Säuglings; er wird durch Wiegen beruhigt und eingeschläfert. Der Korb, die Mulde mit gewölbtem Boden wird auf vielfältige Weise gewiegt: man schwingt sie im Arm haltend, drückt, sie auf eine Bank oder einen Stuhl setzend, wiederholt auf ihren Rand oder stößt sie, wenn sie mit einem Strick von der Decke hängen, immer wieder an. Die Rindenwiege läßt sich nur im Arm oder auf den Rücken gebunden wiegen oder aufgehängt, wie die Tuchwiege, ${ }^{1}$ während die auf dem Gedanken der Unterstützung beruhende Kufenwiege nur durch Drücken oder schwache Stöße auf der runden Kufe schwingt, obwohl es vorkommt, daß sie nach altem Brauch aufgehängt wird. Die Wiegenarten verkörpern also im wesentlichen das Prinzip der Schaukel, aber ihr Schwingprinzip und ihre Funktion sind enger, spezialisierter. Den Zustand von der Funktionstrennung spiegelt der Name der Muldenwiege von Hétfalu hintó (Schaukel) wider.²

Von den als Spielgeräte fungierenden Schaukelarten ${ }^{3}$ für zumindest des Sitzens fähige, meistens aber größere Kinder gibt es hinsichtlich ihres Konstruktions- und Funktionsprinzips grundsätzlich zwei Arten: aufgehängte und unterstützte. In archaischeren Gebieten, so etwa in den Szeklersiedlungen der Bukowina sowie überall

\footnotetext{
${ }^{1}$ Auf den Zusammenhang dieses Gegenstandes mit der Wiege wurden auch der Wiegenforscher HERKELY (1937) sowie K. CSILLÉRY (1982: 31-32, 111-146) aufmerksam, später zitierte ihre Angaben KRISTON (1990: 167). Bei den Tschangos im Burzenland „lag das Kind früher in einer an den Zimmerbalken gebundenen, für diesen Zweck verfertigten Mulde oder einer auf einem Holzrahmen befestigten, aus einem Hanfsack gefertigten hintó, d. h. Schaukel, bzw. csinga ,Schaukel', und man schwang sie an einer Schnur." (SERES 1984: 414).

${ }_{2}^{2}$ Diesem ähnlich bedeutet das rumänische Wort leagăn Wiege und Schaukel gleicherweise.

${ }^{3}$ Diese Studie berücksichtigt nicht die Jahrmarktsschaukeln, sondern nur die „Volksschaukeln“, die selbstgefertigten Gegenstände ähnlicher Funktion und die mit ihnen zusammenhängende Folklore.
} 
in Siebenbürgen, waren diese Gegenstände in selbstgefertigter Form bis in die 1950er Jahre in Gebrauch. ${ }^{4}$

1. Die aufgehängten Schaukeln

Die Spielschaukel ist ein an einem Baum, am Sturzbalken einer Tür oder eines Tores oder an einem selbständigen Gestell mit beiden Enden etwa $40 \mathrm{~cm}$ voneinander entfernt befestigtes Seil oder Kette bzw. einfache oder doppelte Stange, auf denen man sitzend oder stehend schaukeln kann (1a). Der Sitz wird durch einen Sack, ein Brett oder eventuell einen von Leisten eingefaßten Brettsitz bequemer (1b). Die starre Fußschaukel erhält eine Querstange, um stehend darauf zu schaukeln. Auf ihr schaukeln eher erwachsene junge Paare.

In der Ruhestellung befindet sich die Schaukel auf ihrem Tiefpunkt: wird sie ins Schwingen gebracht, schwingt sie vor und zurück, auf und ab, da sich die Schwingkraft mit dem aus dem Eigengewicht ergebenden Beharrungsvermögen summiert. Das Ausschwingen auf der halbkreisförmigen Bahn führt beim Schaukelnden zur Freude an der zyklischen Bewegung, zum Gefühl des Fliegens. Die Schaukel erhebt sich und beschleunigt nach und nach, bis sie den Gipfel erreicht - dessen Höhe auch die Geschicklichkeit und der Mut des Schaukelnden beeinflußt - und dann heruntersinkt und stehenbleibt. Die durch ein Sprüchlein geregelte Dauer des Schaukelns macht dem Schaukelnden die Endlichkeit der angenehmen Dinge bewußt. Das Schwindelgefühl läßt sich durch das Eindrehen des Seiles oder der Kette steigern: Von dem auch um seine eigene Achse kreiselnden Spieler sagt man: „er trinkt Schnaps". 5 Die Jungen können aus Übermut von der schwingenden Schaukel springen: auch dann genießen sie das Erlebnis des Fliegens, wobei sie riskieren, daß sie sich stoßen können.

2. Bei den sich um einen Unterstützungspunkt bewegenden Schaukelarten gibt es zwei Varianten, entsprechend der Bewegungsrichtung:

2a. Die Wippe beruht auf dem Prinzip des zweiarmigen Hebels und bewegt sich rhythmisch auf und nieder. Sie ist im allgemeinen ein horizontal, quer auf einer auf dem Boden liegenden Stange, eventuell einem Klotz balancierendes Brett, das kleinere Kinder selbst oder andere für sie auf dem Hof improvisieren. Die sich selbst abstoßenden Spieler erleben die rhythmische, sich abwechselnd wiederholende Aufund Abbewegung. Wird die Wippe nicht fixiert, kann sie von dem Klotz herabfallen, was die Möglichkeit des Fallens bewußt macht. ${ }^{6}$

2b. Die cserkabala (eine Art von Drehschaukel) ist ein karusselartiges, auf einer stabilen senkrechten Stütze waagerecht angebrachtes, sich im Kreis drehendes Spielgerät. ${ }^{7}$ Auf ihm spielen üblicherweise schon die Halbwüchsigen. Das unterstützende Element, der kabalaláb (Stutenfuß), ,Kabalafuß', ist ein (eventuell absterbender)

\footnotetext{
${ }^{4}$ Heute sind bloß noch die fabrikgefertigten Schaukeln häufig, nicht nur auf den Spielplätzen, sondern auch die Hängeschaukeln in den Türrahmen innerhalb der Wohnblöcke.

${ }^{5}$ KERÉNYI (1957: 43).

${ }^{6}$ Die heutigen Spielplatzwippen sind selbstverständlich fixiert.

${ }^{7}$ Die Quellen der Beschreibung dieses Gegenstandstyps: KRISTÓ (1995: 54-55), des weiteren die Síppal-Dobbal-Sammlung des Szekler Nationalmuseums in Sepsiszentgyörgy 105, 111, 192, 208, 293, 538, $748,788,818,864$.
} 
in ca. 1,50 m Höhe abgeschnittener, am oberen Ende zu einem regelmäßigen Zapfen von 5-6 cm Durchmesser verjüngter Baumstamm mit Wurzeln oder ein in die Erde eingegrabener und festgestampfter Pfosten ${ }^{8}$ mit eingebohrtem Holzzapfen. Fehlt ein solcher, wird der Oberteil mit einem eisernen Nagel befestigt. Der Oberteil ist entweder eine lange Stange (eventuell ein Brett) oder ein Wagenrad.

2b1. Die einer ,Wagenachse ähnliche“ dünne Stange oder das zurechtgeschnitzte Brett, die kabala, wird in der Mitte durchbohrt und an den Enden stattelartig geschnitz, „damit man besser darauf sitzen kann“. Möglichst gleichgewichtige Kinder setzen sich im Reitersitz darauf, damit das Gleichgewicht beider Seiten gewahrt ist. Es kommt vor, daß aus Leisten Sitze auf die Enden des Brettes gebaut werden. Häufig setzen sie sich auf vom Brettende herabhängende Seile oder Ketten, auf die auch ein Brett als Sitz befestigt werden kann. In anderen Fällen dient ein angebundener fogópálca ,Griffstab’ zum Festhalten. Der allgemeinste Name dieses Spiels ist cserkabala.

2b2. Auf den Zapfen des Pfostens wird ein möglichst großes Wagenrad, günstigenfalls mit $1 \mathrm{~m}$ Durchmesser, aufgesetzt: der ördögszekér, ,Teufelswagen', Manchmal wird auf dem Rad noch eine 4-6 m lange Leiter befestigt, auf deren beide Enden sich die Spieler setzen (cserkabala).

Zur Verringerung der Reibung des drehenden Teiles gibt es mehrere Verfahren. Man klemmt in Streifen zwei mit der klebrigen Seite aufeinandergelegte Rindenstücke oder eingefettete Lederstücke darunter oder „schmiert“ mit einem ranzigen Stück Speck, „damit die kabala (Stute) weitergehen kann“; am Nyikó schmiert man das Wagenrad mit Radschlamm ein, ja bringt an der Achse auch den Radtellerring an. ${ }^{9}$

Die cserkabala (Drehschaukel) wird im allgemeinen direkt am Drehelement angetrieben, doch kommt es auch vor, daß man an das eine Ende ein Seil bindet und der Treiber mit ihm im Kreis läuft oder nach jeder Umdrehung der Stange einen Stoß gibt. Um den oberen Teil der Radnabe wickelt man ein Seil: reißt man an diesem, beginnt das Rad sich schnell zu drehen. Dem darauf sitzenden Spieler wird schwindlig, er kann herunterfallen. Bei solchen Gelegenheiten sagt man, „die kabala hat gebockt“. Dem als macsika (unübersetzbar) verspotteten Spieler gießt man Wasser in den Hals. Dieses Spiel ist die Geschicklichkeitsprobe der Halbwüchsigen. „Wer sich zum zweiten Mal draufzusetzen getraut, muß ein Kind mit gutem Nervensystem, gesundem Herz und Magen sein. Die meisten haben Brechreiz, wenn sie glücklich oder herumrollend auf dem Boden ankommen." ${ }^{10}$ Der Spieler muß beweisen, daß er, der Zentrifugalkraft widerstehend, sich sogar bis zur zehnmaligen Umdrehung hinten auf dem Spielgerät halten kann. „Wer die zehn Würfe (Stöße) darauf aus-gesessen hat, hat sich als ganzer Kerl erwiesen“. ${ }^{11}$

\footnotetext{
${ }^{8}$ In Siebenbürgen scheint in der ersten Hälfte des 20. Jh. die sekundäre Form häufiger gewesen zu sein. (Síppal-Dobbal-Sammlung)

${ }^{9}$ KRISTÓ (1995: 55); JóZSEF (1943: 11)

10 JÓZSEF (1943: 11)

${ }^{11}$ KRISTÓ (1995: 54-55)
} 
3. Die allerkomplizierteste, beide Prinzipien vereinende Hängeschaukel, krincsuj oder Schaukelrad „besteht aus zwei Hauptbestandteilen: 1. In die Erde eingegrabenen und auch von der Seite her gestützten Beinen und 2. auf diesen drehbar angebrachten Flügeln, an denen sich Körbe mit Türchen und Sitzen befinden, in der Art, daß diese sich ebenfalls gesondert, um ihre eigene Achse drehen." ${ }^{12}$ Dieses vom Balkan entliehene Gerät bauten die Männer des Dorfes für die Jugendlichen in der Bukowina auf.

\section{DIE SCHAUKEL ALS ZAUBERMITTEL (TRAUMREISE AUF DER SCHAUKEL)}

Die Schaukel wird in der ungarischen Sprache zumeist mit irgendeinem die schwingende Bewegung, das Erlebnis des Schwebens ausdrückenden lautnachahmend-lautmalenden Wort benannt. ${ }^{13}$ Der Name kann auf das Treiben oder im Falle der gestützten Drehschaukeln auch auf die Tatsache des Drehens hinweisen. ${ }^{14}$ Die Drehschaukel wird im überwiegenden Teil der Fälle metaphorisch mit dem weiblichen Pferd assoziiert, diese alte Bedeutung des aus dem Slawischen stammenden Wortes kabola kennt man in Siebenbürgen noch heute vielenorts. In Slawonien wird die Drehschaukel Teufelspferd genannt. ${ }^{15}$ Der Teufel stellt häufig das Attribut in anderen Benennungen dieses Spielgerätes: Teufelsschaukel, Teufelswippe, Teufelshaspel, Teufelsmühle, Teufelsrad und auch Teufelswagen. Seltener läßt das Attribut verrückt oder Glücks- die besondere Rolle des Gerätes ahnen. ${ }^{16}$

Diese volkstümlichen Termini wecken den Verdacht, daß dieses „schleudernde“ oder "drehende“ und zugleich schwindlig machende und betäubende, eventuell in den Traum wiegende Gerät zwischen der wirklichen und der transzendentalen Sphäre vermittelte.

Wie schon angedeutet wurde, hatte das Schaukeln bzw. Drehen als Geschicklichkeitsprobe auch eine Initiationsfunktion: auch das Begießen des Halses des Herunterfallenden erinnert daran. ${ }^{17}$ Die Probe konnte auch auf die Auswahl von Personen mit übernatürlichen Kräften gerichtet sein: einem von JAGIĆ zitierten kroatischen Aberglauben gemäß wurde im Mittelalter der Wetterzauberer (oder der fahrende Schüler) auf der Drehschaukel ausgesucht. Von den die 13. Schule (hier als

\footnotetext{
12 KRISTON (1988: 169) zitiert Imre SZABÓ.

${ }^{13}$ Auf den Zusammenhang mit dem Wortstamm ring ,sich wiegen, schlingern' wurde schon Géza BÁRCZI (1941: 122) aufmerksam. Zitiert von KRISTON (1988: 167).

${ }_{14}$ Áron KISS (1891) nennt es Drehmühle.

${ }_{15}^{15}$ KRISTON (1988: 168)

16 KRISTON (1988: 168); (JÓZSEF 1943: 11).

${ }^{17}$ Bereits beim Schaukeln ohne jedes Hilfsmittel läßt sich die Initiationsfunktion nachweisen: Der Hintern der schwingenden Person wird auf die Erde oder den Balken gestoßen. Das Wesen mehrerer Übertölpelungsspiele besteht darin, daß die Erfahreneren die Erstteilnehmer am Spiel mit Wasser begießen. (GAZDA 1980: 365). Vgl. RÓHEIM (1925: 261).
} 
Klasse) beendenden Kandidaten stellten sich 13 auf ein riesiges Rad mit 13 Speichen, welches in schnelle Drehung gebracht wurde. Wer zuerst vom Rad fiel, der war der Wetterzauberer. ${ }^{18}$

Die Mediatorrolle der Schaukelarten läßt auch der Zeitpunkt ihrer Aufstellung und Benutzung erahnen. József KRISTON Vízi beobachtete, daß die Szekler in der Bukowina sämtliche Schaukelarten am Karfreitag aufstellten: die Wippe für die kleinen Kinder auf dem Hof, die Fußschaukel und cserkabala für die Jugendlichen auf dem gemeinsamen Spielplatz, ${ }^{19}$ die krincsuj vor der Csarda, und Ostern wurde auf ihr erstmals gespielt. ${ }^{20}$ Ähnliche Angaben gibt es auch aus Siebenbürgen. ${ }^{21}$ In Nordungarn wiederum stellte man die Drehschaukel Typ 2b1 zum Spinnsonntag der Fastenzeit her, so daß schon an den Fastensonntagen auf dem Teufelspferd geritten werden konnte. ${ }^{22}$

Während des Osterfestkreises verhalfen mehrere auch durch die Kirche geweihte Gegenstände und Aktionen magisch zur Erneuerung der Natur. Es läßt sich vermuten, daß auch verschiedene Formen des Schaukelns irgendeine magische aber der Aufmerksamkeit der Kirche entgangene - Funktion hatten. Regine Schmidt folgend bringt József KRISTON VízI das Seilschaukeln mit einem antiken griechischen Ritual, der zu Dionysos' Ehren veranstalteten Aiora, in Beziehung. Dabei ,... ließ man Jungfrauen schaukeln und sang festliche Lieder. Dieses Schaukelfest war nach einzelnen Autoren ein Segens- bzw. ein Reinigungs-, nach Nilsson war der Sinn des Schaukelns ein Fruchtbarkeitsritual ..."23 Die Vermutung des englischen Verfassers akzeptiert auch Philippe Aries: „Die Schaukel war auch noch im 18. Jahrhundert eines der am häufigsten abgebildeten Spiele, sie war ein rituelles Element eines Kalenderfestes, der Aiora, des Festes der Jugend: Die Jungen sprangen auf einem mit Wein gefüllten $\mathrm{Fa} ß$ herum und ließen die Mädchen schaukeln. Später verlor das Spiel, das sich von der religiösen Symbolik getrennt hatte, seinen Gemeinschaftscharakter, wurde weltlich und individuell.“"24

Die einstige Ritualfunktion der Schaukel lassen auch einige Textmotive der ungarischen Schaukelsprüche vermuten, die die Schaukeldauer der Spieler messen. ${ }^{25}$

\footnotetext{
18 RÓHEIM (1925: 35). Auf die Angabe machte mich István Pál DEMÉNY aufmerksam.

${ }^{19}$ Der Spielplatz der Jugendlichen an Fest- und Sonntagen war am Nyikó im Scheunengarten (JÓZSEF 1943: 11). Eine gemeinsame Schaukel- und Spielgelegenheit bot außerdem das Hüten der Jungen am Dorfrand, auf den Waldweiden (KRISTÓ 1955: 55).

${ }^{20}$ KRISTON (1988: 169)

${ }^{21}$ Síppal-Dobbal-Sammlung, 818. KISS (1891) nennt das gleiche Datum des Beginns.

${ }^{22}$ KERÉNYI (1957: 16). ÚMTSz II, 715, Stichwort guzsalyhinta. Spinnsonntag wurde der zweite bzw. vierte Fastensonntag genannt, an dem das Weben und Spinnen eingestellt wurde (BÁLINT 1976: 190, 192).

Regine SCHMIDT zitiert von KRISTON (1988: 170).

24 Zitiert von KRISTON (1988: 170).

${ }^{25}$ Die Basis meiner Beobachtung ist die Analyse von 276 als Schaukelsprüche publizierten Texten im Computerprogramm ISIS 3.0. Den in meinem Material 67mal vorkommenden Typ Egy, megérett a meggy (MNK 2010/2) sowie eine Variante des sich als Kettenmärchen fortsetzen lassenden Tíz, tiszta víz (MNK 2018A1*) - bei mir 26 Varianten -, außerdem den mit Adjál, anyám, vékát beginnenden Kettentext (MNK 2018A2*) - bei mir 10 Varianten - qualifizierte Ágnes KoVÁCS als Formelmärchen und veröffentlichte ihre Bibliographie in Band 9 des Ungarischen Märchenkataloges. (KovÁCS-BENEDEK 1990: 49-56, 84-88).
} 
Die Texte dieser Gattung ${ }^{26}$ sind nach ihrer Herkunft und ihrem Charakter vielfältig: Im Wechsel mit von Erwachsenen geschaffenen Lehrdichtungen ${ }^{27}$ kommen ganz verschiedene, zumeist bruchstückhafte, durch und durch kontaminierte und deshalb sinnlos scheinende, aus den unterschiedlichsten Zeiten stammende gereimte Texte vor. Ihre heutige Funktion, das Auszählen, stört ihre Sinnlosigkeit nämlich nicht, viel eher ihre Deutung. ${ }^{28}$

Unter den hervorgehobenen Tagen berufen sich die Sprüche niemals auf Ostern, viel eher auf Weihnachten (11). ${ }^{29}$ Brauchen vielleicht die Tierjungen (13) - die Sau hat geferkelt, dreiwöchig ist das Ferkel (4) - die Erwärmung des Wetters? Die Lerche kündigt den Frühling an, eventuell treibt sie ihn an (9).

In mehreren Texten ist von der Reise die Rede, was in Kenntnis des Schaukelpferds bzw. Wagens auch natürlich ist. Der Assoziation von Schaukel und Pferd verdankt auch das Schaukelpferd seine Existenz. ${ }^{30}$ Der Erwachsene kann das Kind auch ohne Gerät, auf den Knien, reiten lassen. ${ }^{31}$ Selten identifizieren die Sprüche die Schaukel mit dem Pferd, z. B. Steig ab, Mönch, vom Pferd! (1). Häufiger verweisen sie auf das Reisen mit dem Wagen bzw. der Kutsche und auf den Umstand, daß die Räder geschmiert werden müssen (33). ${ }^{32}$ Eine Reihe von Kettenhandlungen ist zur Beschaffung des Schmers nötig: Gartenbearbeitung, Kürbispflanzen und -begießen, Schweinemästen.

Häufig sprechen die Texte vom Hinaufreisen, Aufsteigen. Das ausgesprochene Ziel der sich anstrengenden Spieler - Treib bis zum Ende, nicht nur halb (2) - war das (dreimalige) Anstoßen am Unterzug (5), das Erreichen der Lerche (4). Für das Erreichen des Unterzuges kann der Spieler ein rotes Ei bekommen oder einen roten

\footnotetext{
${ }^{26}$ Auch die Frage, ob es sich um eine Gattung handelt, ist bestreitbar: Von den analysierten 276 Texten sind nämlich 13 erkennbar von anderer Gattung, sicher wurde nur zufällig auf ihren Text geschaukelt. Andererseits kommen die Schaukelsprüche oder Fragmente von ihnen auch in anderen Gattungen vor, z. B. in Spott- und Auszählversen usw.

${ }^{27}$ Diese beginnen am häufigsten mit der Textformel hinta-palinta. Im untersuchten Material gehören rund 100 Texte zu dieser Kategorie, und weitere 10 sind anders aufgebaute Stücke. 20 von den ersteren teilen nur die Tatsache des Schaukelns des Kindes mit: Es schaukelt das Baby (Jancsika) (im weiteren die Zahl der Texte kurz in Klammern). Vermutlich sind die die Lerche erwähnenden Sprüche Schöpfun-gen von Kindergärtnerinnen (9): z. B. Wir fliegen wie die Lerche, sowie jene, die das Attribut Weihnach-ten enthalten: z. B. kleine Weihnachtsschaukel (4) oder Weihnachts-Katica (4) oder Weihnachtstier (2), Krümel (1). Trotz ihres künstlichen Stils fügen sich diese Sprüche zum Teil in das Paradigma ein: sie enthalten Motive, die sich auch in den übrigen Texten finden. Die gleiche Anfangsformel ist glaubwürdig, sie führt auch ins magische Substrat gehörende Texte ein: z. B. Alte Donau, kleiner Soldat, spring in die Donau (14).

${ }^{28}$ Bei der Deutung übernehme ich nicht die in der Ethnologie allgemein akzeptierten Feststellungen, sondern versuche die Entdeckung der Bedeutungen aufgrund der ungarischen Folklore. Da mir allerdings kaum Vorarbeiten zur Verfügung stehen, deutet meine Arbeit die Probleme eher nur an, als daß sie sie löst.

${ }^{29}$ Siehe Anm. 27.

${ }^{30}$ Die Figuren der Karussells des Unterhaltungsgewerbes sind ebenfalls entweder Pferde oder Verkehrsmittel - heute Autos.

${ }^{31}$ Damit hängen die Sprüche beim Reitenlassen auf den Knien vom Typ Hü, mein Pferdchen, nach Barassó zusammen.

${ }^{32}$ Die Popularität dieses Texttyps kann mit der schon vorgestellten Weise erklärt werden, das Rad funktionsfähig zu machen.
} 
Apfel oder Maiskolben abreißen (1-1). ${ }^{33}$ Andere mögliche, sich steigernde Obergrenzen sind der Hahnenbalken (12), die Spitze des Nußbaums (1), der Turm meines Großvaters (1), die Dornspitze (1), das (Roten-) Turmdach (17) bzw. die Mitte des Himmels, der Sternhimmel (11), das Haus Gottes (1). Denn von hier aus kann man sehen, was Gottes Kuh gekalbt hat. Nach einigen Texten gelangt der Schaukelnde also bis in die jenseits der Erfahrungen befindliche Oberwelt. Eine Voraussetzung dafür ist der Traum (1 Spruch und 2 ungereimte Dialoge weisen darauf hin), und in den Traum wird man durch die Schaukel versetzt, und zwar erst nach einer gewissen Anzahl von Schwüngen.

Es hat den Anschein, daß das Maß des Schaukelns nicht willkürlich bestimmt wurde: Den Texten gemäß tritt nach einer bestimmten Zahl von Schwüngen, seltener nach drei, sechs, neun, zwölf, am häufigsten nach zehn Schwüngen, eine unerwartete Wende ein.

Nach einem selteneren Typ (9) dreht irgendjemand um, eine ausgeraste Person: unsere Mutter Hebamme, die Tochter der kleinen Königin, das Königstöchterchen (je 1) oder die Singenden. Ein (mythisches?) Tier wird geboren: Die kleine Kuh Gottes (der Königin, unserer Mutter Hebamme) hat etwas Buntscheckiges gekalbt. Zwei Texte sprechen gute Wünsche aus, einer in der Art eines Spielmannsliedes: Behüt ihn Gott auch weiter, Mit hundert Wagen Heu, Mit hundert Eimern Wasser. Dieser Texttyp betont auch, daß wir uns auf einem absonderlichen Schauplatz befinden: im Gyúröser oder im Bélágyer Garten, bzw. im Garten von Richters, wo phantastische Dinge passieren: Elstern zanken sich, eine von ihnen sticht der Adler, die Grillenmutter oder Frau Richter ab. „Ein Tropfen Blut tropfte, in Debrecen würde er nicht hineinpassen, das Goldbrett würde spalten, da würde er hineinpassen. "Biblische Gäste treffen ein, für sie wird das Festmahl zubereitet: Sankt Peter und Sankt Paul, galloppieren auf grauem Gaul, der frißt winziges Heu, scheißt schwarzes Pech, Damit macht man Licht. (4)

Ein wichtiges, Wenden vorbereitendes, häufiger das Zählen abschließendes, seltener selbständiges Motiv ist das Wasser. Zuweilen erscheint es auch im Zusammenhang mit der Erfahrungswelt: Hemd und Hose des Schaukelnden sind ganz naß (2), seine Gefährten begießen seinen Hals aus dem Turm (1), seine Schwägerin badet ihn (1), viel beachtenswerter ist aber seine symbolische Rolle. ${ }^{34}$ Nicht so interessant ist die seltene Hinzufügung, daß den Schaukelnden der Teufel ins Wasser bringt (2). Wichtiger ist die Klassifizierung, daß sauberes und nicht sauberes Wasser unterschieden werden. Letzteres ist für Tiere bestimmt: Wenns nicht sauber ist, brings zurück, Dort ist das Eselchen (Esel, Hahn, Ferkel), das wirds trinken (28). Das Flußwasser ist sauber, genauso wie das im Trog, im Eimer befindliche, in ihn hineinflieBende warme Wasser. Den schaukelnden Jungen - kleinen Soldaten - ermuntert man zum Sprung in die Donau oder Theiß (14). Das Mädchen: das Mädchen meiner Schwägerin, Gevatterin bzw. meiner Frau Mutter (9), metaphorisch eine Malve (1), ein Wels (1), eine Katze (3) oder einfach die Braut (6), manchmal ein Bursche, eine Frau,

\footnotetext{
${ }^{33}$ Im Zusammenhang mit dem Apfel s. Anm. 36. Der rote Maiskolben ist das Vorzeichen des Hochzeitsmahles. Das rote Ei vereint von Ostergeschenk bis zum Auferstehungssymbol viele Bedeutungen in sich.

${ }^{34}$ Der realen Grundlage des Naßmachens begegneten wir schon bei Behandlung des Schaukeltyps $2 \mathrm{~b} 1$.
} 
in einem Fall die Großmutter fiel in die, ertrank, starb (27) in der Theiß, Donau (9), im Brunnen, Trog oder in 3 Eimern warmem Wasser bzw. im Honigfaß. Die äußersten - parodistischen - Texte verweisen vielleicht darauf, daß man die Tatsache des Todes nicht ernst nehmen muß. Die Gemeinschaft geht nach, zieht heraus, schleppt heraus, fischt heraus, schöpft aus. Bei der Rettung helfen kann das Bettlaken, Siedentuch (4), 12 Mücken, 13 Flöhe, 14 Zecken (1) oder aber Lärm, Musik: Pfeife, Trommel (Maultrommel), feine (Geigen)musik (6). Man läutet für ihn mit zwei leeren Kannen (2), Henkelkrügen, dem Aneinanderstoßen von Weinfässern (1-1), Tonscherben, Knochen (9), Eisenstücken (1) oder zieht ihn heraus. Die Musik ist auch ein Mittel zum Herbeiholen des Vögelchens im grünen Wald (1).

Eine Deutung erfordernde Elemente sind das Ins-Wasser-Springen, Im-WasserSterben, Retten bzw. die Hilfsmittel dazu. Nach dem Zeugnis der ungarischen Volkslieder treibt Hochwasser, Fließwasser die Mädchen fort, und die Burschen holen das ihrige heraus. Also ist das Ins-Wasser-Fallen/Retten ein Liebes-/Hochzeits-symbol. ${ }^{35}$ Eines der beliebten Partnerwahlspiele der Jugend ist Ich bin in den Brunnen gefallen, wer zieht mich heraus? Das Wesen des Spiels ist das Herausziehen durch einen Kuß. Bettlaken und Seidentuch sind Hochzeitszubehör: Wird eine Frau mit ihnen herausgezogen, ist das Laken voll Löcher und das Tuch gelb (1). Die ungarische Fachliteratur hält das Heilen mit Musik für ursprünglich schamanistisch, ${ }^{36}$ auf jeden Fall ist die Musik auch ein Bestandteil der Hochzeitszeremonie. Die Lärmerei wiederum hat die Funktion, Böses abzuwenden. Und was das parodistische Retten mittels Mücken, Flöhen und Zecken betrifft, so weist der ganze Text auf sein Verständnis als Fiktion, als Scherz hin.

Jenseits des Wassers (in einer anderen Welt?) wohnt eine seltsame Figur, ein Mönch (Fräulein), der nichts von seiner gebratenen Wurst abgibt (5). Ein Zubehör dieses wegen seiner Unvollständigkeit allein nicht zu interpretierenden Texttyps ist die Opposition Fleischgericht/Nudelgericht: man schneidet es mit dem Beil ab und bringt dann das blutige (nach 2 Texten Katzen-) Fleisch in einem überdeckten Korb auf den Markt, lügt aber, es seien Nudeln, Maultaschen. Auch gesondert kommt sowohl das Nudelgericht (7) als auch das Fleischgericht (3) vor. Sie sind wahrscheinlich Sexualsymbole. Bei ihrer Identifizierung können andere Gattungen helfen, so die Spottverse, Rätselfragen sowie die Vermählsprüche. ${ }^{37}$

\footnotetext{
${ }^{35}$ Darüber im einzelnen: DEMÉNY (1997). Ein allgemein bekanntes ungarisches Volkslied drückt das so aus: Vor meinem Tor ein Fischteich, Fischteich. / Ich fiel hinein mit Wagen und Pferd. / Ach, Gott, wer zieht mich heraus, ach, wer zieht mich heraus? / Tut jemand noch mein Hemd leid? /Ich zieh dich raus, süßes Püppchen, raus, raus, raus! / Ich laß dich nicht im Fischteich sterben. / Ich verkaufe mein Braut-tuch, meinen Ring, / Ich löse meinen liebsten Liebsten aus. Mskr. im Folklore-Archiv Klausenburg FM 724. Sammlung von János JAGAMAS.

${ }_{37}^{36}$ RÓHEIM (1912)

${ }^{37}$ Darauf verweist z. B. die folgende Rätselfrage bzw. ihre Auflösung: Es wird auf den Rücken gelegt und mit rohem Fleisch gepatscht. Was ist das? (Nudelteigkneten) 1990 zitierten auf der Eisenbahn pendelnde Arbeiter aus Kreis Kovászna beim Klatsch über irgendjemand mit hämischem Lächeln den Text, den ich immer für einen Kinderspruch gehalten hatte: Ich sage nichts, du sagst auch nichts, und die Katze hat die ganze Milch aufgeschleckt. Eine ähnliche Deutung des Nudelteigs erlaubt der folgende Volksliedtext aus dem Klausenburger Folklore-Archiv: Auf dem Tisch der Nudelteig, Die Katze sprang hinein Schuld daran hat nicht die Katze, Die Hausfrau war betrunken (FM 588).
} 
Die Beziehung zwischen dem Schaukeln und einerseits der Kommunikation mit Gott, andererseits der Heirat als schicksalsentscheidende Lebenswende beleuchten weitere Sprüche. Das Ziel der horizontal (irdisch) gerichteten Reise ist die Fahrt auf den Markt, in die Stadt (z. B. nach Großwardein) (17), um dort ein schönes Kleid zu kaufen (17), (das weiße Kleid wird in sauberem Wasser ausgewaschen), das Tanzen darin (2) oder in die Kirche zu gehen, eventuell Kirchenbau, Anbetung Gottes (13). Dafür muß der Wagen geschmiert werden, wie ebenso dafür, daß man Mädchen, Frauen, Puppen (des weiteren Zigeunerkinder) kutschieren kann: Diese Frauen werden später die Lumpenflickerinnen, Kükenerzieherinnen (7). Ein anderer Zweck des Wagenschmierens ist der Materialtransport: Bretter, Steine und Ziegel, aus denen man ein Haus baut (7), in das schöne Jungen und Mädchen verheiratet werden (9).

Beachtenswert ist, daß im Anfangsteil der kettenmärchenartigen Sprüche der Goldapfel, Flaschen(Hut)apfel, die Truhe der Schwägerin Gegenstände des Auszählens sind. Apfel ${ }^{38}$ wie Truhe ${ }^{39}$ sind Symbole für das Mädchen, die Truhe selbst ist auch der Inbegriff der Aussteuer des Mädchens. Die Anfangsformel eines anderen Auszähltyps, die Sauerkirsche ist gereift, ist ebenfalls ein auf das Aufwachsen des Mädchens verweisendes Symbol. ${ }^{40}$ Den rumänischen Kolindern gemäß wartet das große Mädchen im Obstgarten, z. B. im Sauerkirschgarten, eventuell in einer am Kirschbaum (Fichte) angebundenen Seidenwiege stickend auf ihren Freier ${ }^{41}$ Einem anderen Schaukel-Kettenmärchen (10) gemäß schöpft die Mutter die Perlen mit dem Scheffel, daraus fädeln sie einen Garten auf, in dem die Blumen für den Hut der Burschen und den Busen der Mädchen gezogen werden. Für das Mädchen ist die Rose, für den Burschen der Strau $\beta$ - besagt der auch in die Schaukelsprüche gelangte Volksliedtext (1). Der Strauß ist das Symbol der Liebe: für seine Übergabe und Tracht gibt es einen ausgearbeiteten Ritus im ungarischen Volksleben. Die im Garten gezogene Blume in den ungarischen Volksliedern ist das Symbol des unter dem Schutz der Eltern stehenden Mädchens. ${ }^{42}$ Den Schaukelsprüchen gemäß neigt sich der Zweig der im Rosengarten gezogenen roten Rose und weißen Rose, wenn der kleine Soldat in die Donau springt (2).

Die Schaukel führt also den Schaukelnden aus der Alltagswelt in die Welt der Träume. Letztlich sind diese Träume sogar sehr irdisch: sie hängen mit der Fruchtbarkeit der Tiere bzw. der Verheiratung der Jugendlichen zusammen, nur zu ihrer Erfüllung brauchen sie himmlische (göttliche, aber wenn wir auch den Namen der

\footnotetext{
38 Über die Apfelsymbolik in ungarischen Volksliedern s. DANKÓ (1963).

${ }^{39}$ Gábor LÜKÖ (1990: 36-38)

${ }^{40}$ Das runde Obst ist allgemein ein Symbol des Mädchens. Nicht auszuschließen ist, daß in diesen Spruchtypen mehrere, sich auf andere Zahlen reimende Formeln ebenfalls symbolische Bedeutungen hat-ten. Da die Spieler dies schon recht lange Zeit nicht mehr verstanden, formulierten sie es so um, daß ihre Interpretation den heutigen Forscher vor große Schwierigkeiten stellte. In dieser Studie wird auf ihre ausführliche Erörterung verzichtet.

41 DEMÉNY (1997: 307). In 17 von 46 Texten hängt die Wiege an einem Baumast, in 10 steht sie in einem Obstgarten und einmal hängt sie vom Himmel herab.

42 „Gar nicht das ist die Rose, Die sich im Garten öffnet, Sondern das ist die Rose, Die einander lieben."
} 
Schaukel berücksichtigen, fallweise teuflische $)^{43}$ Hilfe. Die Texte nehmen magisch die Verheißung irdischen Glücks voraus.

Dieses Zukunftbild allerdings ist traumhaft: Diese Dichtung kennt jene Tiefenschichten des Unerbewußtseins, gemäß denen wir durch die Beendigung unseres momentanen Zustandes, durch symbolischen Tod und Auferstehung, in einen anderen Zustand gelangen.

Mit dem Ende des Schaukelns endet auch der Zauber: Der Hahn wird vom Sitz verjagt und zum Aufwachen bietet man ihm Wasser an: Husch, Hahn, runter vom Sitz, trink einen Schluck aus dem Eimer (aus der Tasse) (11). Die Zeit der Partnerwahl ist noch nicht gekommen, schon deshalb braucht er Wasser. ${ }^{44}$ Oder der Grund ist, daß die Braut/der Bräutigam noch nicht die/der Seine/Ihre ist: Der Schaukelnde schaukelt nicht für sein eigenes Geld (13). ${ }^{45}$

Ähnlichen Vorstellungen begegnen wir in mehrerer Hinsicht in der Folklore der in Zeit und Raum fernstehenden Keten (Jenissei-Ostjaken) und Juraksamojeden. Die Mythologie der sibirischen Keten spricht dem Wiegen auf der Schaukel und in der Wiege eine ins Leben und in den Himmel bringende Funktion zu. In der am Schamanenstab aufgehängten Wiege wiegt sich der Held des Mythos (kinderlose alte Männer schaffen sich mittels Wiegens von unbelebten Stoffen - Gänsemist, Tonpuppe, Tonkrüge - in der Wiege Kinder). „Wenn diese sterben, erwecken sie sie durch Wiegen wieder ins Leben. Über den Sohn der Erde (Banrexyp) sagt der Mythos, daß ihm, als die Tochter des Himmels ihn verließ und mit ihr ihn seine Kraft verließ, daß er fast schon starb, der Himmel (Es - Gott) eine Schaukel gab [...] und verfügte, daß er auf ihr schaukeln solle. Damit gewann der Sohn der Erde seine Kraft wieder und konnte sich in den Himmel erheben, wohin seine Ehefrau sich entfernt hatte. Seither hat der Sohn der Erde, der in der zweiten Ebene des siebenstöckigen Himmels ist, immer eine Schaukel (devj-oks). Die Jenissei-Ostjaken stellten diese Schaukel symbolisch mit jener Schaukel dar, die Ende März - in ihrer Sprache: qajetin („der Monat des Elches“) - beim mit dem Ende der Jagd auf die Großhufer stattfindenden Frühlingsfest aufgestellt wurde. Die Männer banden ihre Schaukel an den Stamm zweier nebeneinanderstehender Tannen, die Schaukel bewegte sich wie ein Pendel. Am Morgen, bei Sonnenaufgang mußte man mit dem Gesicht nach Osten auf ihr schaukeln. Das Recht des ersten Schaukelns gebührte dem Schamanen." ${ }^{46}$ IVANOV interpretiert die Wiege und das Wiegen des Kindes darin als die zweite Geburt des Mtyhoshelden, als ein sein Schamanwerden symbolisierendes Zeichen.

\footnotetext{
${ }^{43}$ Es ist nicht klar, ob nicht auch der folgende Text solchen Bezug hat: Dort kommt ein gelbes Fohlen (1), eine alte Frau (1), setz dich auf dessen/deren Rücken! (1-1).

${ }^{44}$ Wenn ein zu junges Bürschchen in Esztelnek wagte, eine Visite zu machen, bot man ihm zur Schande etwas an, drückte ihm Essen oder eine Tasse Wasser in die Hand und verspottete ihn auch mündlich (GAZDA 1980: 121).

${ }^{45}$ Möglicherweise formuliert folgender Text ein Tabu: Hase, Hase, kahler Hase, soll seine Braut kahl werden, die ihn anfaßt.

${ }^{46}$ IVANOV (1984: 218-220)
} 
Die (Eisen-) Schaukel ist in der juraksamojedischen Folklore eher ein gegnerisches Gerät: Man kann in ihr gesund werden, ${ }^{47}$ aber da sich neben ihr der Niedergang in die Unterwelt befindet, kann man auch dahin gelangen. ${ }^{48}$ Am jenseitigen Meeresufer schaukeln 14 Riesen einen Menschen auf der Eisenschaukel. ${ }^{49}$ Als Probe vor der Heirat wollen sie den Helden auf die Schaukel setzen, der sie aus Protest zerstört. ${ }^{50}$

In der Mythologie der Keten hängt also die Schaukel ebenfalls mit dem Vorstellungskreis von Tod und Auferstehung und der Wiederbelebung der Natur, dem Frühlingsfest, in der Folklore der Samojeden dagegen mit dem Wasser, dem Tod bzw. der Heirat sowie der Erprobung zusammen. Die Aiora war, wie zitiert, ein Reinigungs-, Segens- und Fruchtbarkeitsritual der Jugend. Alle diese Elemente lassen sich auch in der ungarischen Folklore nachweisen.

Diese komplexe mediatorische Funktion füllt die Schaukel seit langem (nach dem 18. Jahrhundert?) nicht mehr aus. Vielleicht wurden die rituellen Texte deshalb weniger (die interessantesten wurden am Ende des vergangenen bzw. in der ersten Hälfte unseres Jahrhunderts herausgegeben), ${ }^{51}$ und vielleicht lassen sich damit ihre parodistischen Elemente erklären. Dann, mit dem Verblassen ihrer ursprünglichen Bedeutung konnten sie deshalb mit Textteilen anderer Natur kontaminiert werden, fallweise mit den Schöpfungen bekannter Dichter. ${ }^{52}$ Die Gattung blieb allerdings bis in unsere Tage erhalten. ${ }^{53}$ Erhalten blieb in ihr die Metapher der Reise, wenn auch unter die klassischen Verkehrsmittel die Rakete und unter die Reiseziele der Kindergarten- und Schulweg sowie das Lernen von Gedichtchen aufgenommen wurde.

\section{LITERATUR}

BAKos, József

1953: Mátyusföldi gyermekjátékok (Kinderspiele aus dem Mátyusföld). Budapest.

BÁLINT, Sándor

1976: Karácsony, húsvét, pünkösd. A nagyünnepek hazai és közép-európai világából (Weihnachten, Ostern, Pfingsten. Die hohen Festen aus der ungarischen und mitteleuropäischen Welt). Budapest.

BÁRCZY, Géza

1941: Magyar Szófejtő Szótár. Budapest.

BORSAI, Ilona-HAJDÚ, Gyula-IGAZ, Mária

1977: Magyar népi gyermekjátékok (Ungarische Kinderspiele des Volkes). Budapest.

BORSAI, Ilona-KOVÁCS, Ágnes

1975: Cinege, cinege, kismadár (Meise, Meise, Vögelchen). Budapest.

${ }^{47}$ LeHTiSALO (1947: Nr. 56.)

${ }^{48}$ CASTRÉN (1940: Nr. 5.)

${ }^{49}$ In einem szudbabc (Heldenlied alten Stils). Der Held zerstört die Schaukel (CASTRÉN 1940: Nr. 4).

${ }^{50}$ In einem jarabc (Heldenlied neueren Stils). MiKUSEV (1969: 169-217.). Für sämtliche samojedische Angaben ist István Pál DEMÉNY zu danken.

51 KISS (1891); GÁSPÁR (1977); KERÉNYI (1957); BAKOS (1953)

52 FARAGÓ-FÁBIÁN (1982: 18)

531976 wurden zur Ausschreibung einer Biharer Zeitung 142 Schaukelsprüche eingesandt. FARAGÓFÁBIÁN (1982: 97-113) 
CAstrén, M. A.

1940: Samojedische Volksdichtung. Mémoires de la Société Finno-Ougrienne 83. Helsinki.

CSETE, Balázs

1993: A jászkiséri gyermek élete a születéstôl a házasságig (Das Leben des Kindes von Jászkisér von der Geburt bis zur Heirat). (1954) Szolnok.

K. CSILLÉRY, Klára

1982: A magyar népi lakáskultúra kialakulásának kezdetei (Die Anfänge der Entstehung der ungarischen Wohnungskultur des Volkes). Budapest.

DANKÓ, Imre

1962: Az almaszimbolika magyar vonatkozásai (Die ungarischen Bezüge der Apfelsymbolik). Ethnographia LXXIII, 558-589.

DEMÉNY, István Pál

1997: Trecerea peste apă în folclorul românesc şi maghiar. Anuarul Arhivei de Folclor XV-XVII, 301-317.

FARAGÓ, József-FÁBIÁN, Imre

1982: Bihari gyermekmondókák (Biharer Kindersprüche). Bukarest.

GÁGYOR, József

1982: Megy a gyưrú vándorútra. Gyermekjátékok és mondókák (Der Ring geht auf Wanderschaft.

GÁsPÁR, János Kinderspiele und Sprüche). Band I, Bratislava.

1976: Kerekecske dombocska (Sprüchleinen für Kinder). Dritte Ausgabe. Bukarest.

GAZDA, Klára

1971: Játszani is engedd ... fiadat (Laß deinen ... Sohn auch spielen). Megyei Tükör, 8. Aug.

1980: Gyermekvilág Esztelneken (Kinderwelt in Esztelnek). Bukarest.

HERKELY, Károly

1937: Bölcsőtípusok hazánkban (Wiegentypen in Ungarn). Néprajzi Értesító XXIX.

HiNTALAN, László-LÁzÁR, Katalin

1980: Gyermekjátékok (Kinderspiele). Hévizgyörk. Szentendre.

IVANOV, V. V.

1983: Obi-ugor és ket folklórkapcsolatok (Obugrische und ketische Folklorebeziehungen). In: Nyelv, mítosz, kultúra. Budapest, 215-233.

JóZSEF, Dezsó

1943: A Nyikó-menti gyermek magakészítette játékszerei (Die selbstgefertigten Spielgeräte der Kinder am Nyikó). Kolozsvár.

KÁLMÁNY, Lajos

1877: Koszorúk az Alföld vad virágaiból. I. Pécskáról való (Kränze aus wildwachsenden Blumen der Tiefebene. I. Von Pécska). Arad.

1878: Koszorúk az Alföld vad virágából. II. Elegyes (Kränze aus wildwachsenden Blumen der Tiefebene. II. Elegyes). Arad.

1882: Temesköz népköltése (Volksdichtung aus dem Temesköz). II. Arad.

KERÉNYI, György

1957: Gyermekjátékok (Kinderspiele). A Magyar Népzene Tára I (2. Ausgabe). Budapest.

KovÁcs, Ágnes-BENEDEK, Katalin

1990: A magyar formulamesék katalógusa (Katalog der ungarischen Formelmärchen). Magyar Nép-

KISS, Áron mesekatalógus IX. Budapest.

1891: Magyar gyermekjáték-gyújtemény (Ungarische Kinderspielsammlung). Budapest.

KRISTON VÍZi József

1988: Hintaformák és a hintázás szokásai Közép-Kelet-Európában. In: Múzeumi Kutatások BácsKiskun Megyében (Museumsforschungen im Komitat Bács-Kiskun). Kecskemét 1988. 166172.

KRISTÓ, Tibor

1995: Cserkabala. In: KRISTÓ, Tibor, ed: Csíki néprajzi gyưjtések. Csíkszereda. 54-55.

KRIZA, János

1911: Vadrózsák. Székely népköltési gyújtemény (Heckenrosen. Szekler Volksdichtungssammlung). Band I. Budapest. MNGY XI.

KRIZA, János-ORBÁN, Balázs-BENEDEK, Elek-SEBESI, Jób

1882: Székelyföldi gyújtés (Sammlung aus dem Szeklerland). MNGY III. 
LÁBADINÉ KEDVES, Klára

1989: Gyingyet-gyöngyöt asszonyának. Drávaszögi (alfalusi) népi mondókák és gyermekjátékok. (Volkssprüche und Kinderspiele aus dem Drauwinkel, Alfalu). Eszék.

LEHTISALO, T.

1947: Juraksamojedische Volksdichtung. Helsinki. Mémoires de la Société Finno-Ougrienne 90.

LÜKŐ, Gábor

1990: Anya- és csecsemőóvó képek a régi magyar szekrényeken (Mutter- und Säulingsschützbilder in alten ungarischen Schränken). In: Gyökereink 2, 11-53.

MATIJEVICS, Lajos

1977: Tíz, tíz, tiszta víz. Jugoszláviai magyar népi mondókák (Zehn, zehn, reines Wasser. Jugoslawisch-ungarische Volkssprüche). Újvidék.

MIKUSEV, A. K.

1969: Komi epiceskie pesnyi i ballady. Leningrad.

RóHEIM, Géza

1912: Sámánkodó gyógyítás nyoma egy gyermekversben (Spuren schamanenartiger Heilung in einem Kindervers). Ethnographia XXIII, 360-362.

1925: Magyar néphit és népszokások (Ungarischer Volksglaube und Volksbräuche). Budapest.

SERES, András

1985: Barcasági magyar népköltészet és népszokások (Ungarische Volksdichtung und Volksbräuche aus dem Burzenland). Bukarest.

SZABÓ, Imre

1906: Farsang és húsvét a dévai csángó telepeken (Fasching und Ostern in Tschango-Siedlungen von Diemrich). Néprajzi Értesítő VII, 92-93.

TÖRÖK, Károly

1872: Csongrádmegyei gyújtés (Sammlung im Komitat Csongrád). Pest. MNGY II.

ÚMTSz.

1979: Új Magyar Tájszótár (Neues Ungarisches Dialektwörterbuch). Hrsg. B. LôRINCZY, Éva. 